\title{
Compromiso cardíaco en pacientes con enfermedad de Fabry
}

María Griselda Gómez, Carmen Varas, Miguel Morales, Fernando Bonacic, Mariela Álvarez ${ }^{1}$, Ana Rojas².

Hospital San Pablo, Coquimbo, Chile.

(1) Centro de Especialidades Médicas Coquimbo (CEMCO).

(2) TPM: técnico paramédico Hospital San Pablo, Coquimbo

Antecedentes: La enfermedad de Fabry (EF) es un desorden lisosomal de transmisión ligada al cromosoma $\mathrm{X}$ debido al déficit de la enzima alfa galactosidasa A, con acumulación multisistémica de globotriaosilceramida (GB3). La afectación cardíaca reduce expectativa y calidad de vida.

Objetivo: Describir compromiso cardiológico de 38 pacientes con $\mathrm{EF}$, diagnosticados y estudiados multidisciplinariamente. Destacar alta prevalencia en esta región.

Método: A partir de caso índice, se aplica encuesta y elabora familiograma de 5 familias. Estudio genético y enzimático de 65 sospechosos confirma 38 afectados (25 Mujeres y 13 hombres) evaluados multidisciplinariamente con Electrocardiograma, Ecocardiograma y exámenes de laboratorio.

Resultado: Compromiso cardiológico en $66 \%$ de adultos, no presente en niños. Cardiopatía hipertrófica $(\mathrm{CH})$ fue el hallazgo cardiológico más frecuente
(56\% de adultos) presente en $63 \%$ de los hombres y en $52 \%$ de las mujeres. En mayores de 40 años, $100 \%$ de hombres y $82 \%$ de mujeres están afectados. La edad promedio fue 38 en hombres y 57 en mujeres. Cardiopatía dilatada $26 \%$ en su mayoría asociado a CH. Insuficiencia valvular mitral leve en $47 \%$, con predominio femenino, PR corto en 7 mujeres, fibrilación auricular 2 mujeres y 1 hombre, TPSV 1 mujer, BAV completo 1 hombre. Sin eventos coronarios.

Conclusión: La afectación cardíaca en nuestro grupo es similar a la reportada internacionalmente. Dada la alta prevalencia que tiene esta patología en nuestro medio, frente a un paciente no hipertenso con hipertrofia ventricular debería descartarse EF. Evaluación dermatológica y oftalmológica apoyaría diagnóstico presuntivo antes de confirmación enzimática o estudio genético.

Palabras claves: Enfermedad de Fabry, variante cardíaca, genética.

\section{Correspondencia:}

Dra. María Griselda Gómez:

Marbella 5008. Jardines de Peñuelas,

Coquimbo.

griseldamg@gmail.com . 


\section{Cardiac involvement in patients with Fabry's Disease}

Fabry's disease (FD) is a lysosomal disorder with an $\mathrm{X}$ chromosome linkage. It is related to a deficiency of alpha-galactosidase A, leading to multisystemic accumulation of globotriasocyl ceramyde (GB3). Cardiac compromise reduces life expectancy. Herein we describe cardiac and systemic findings in 38 patients with FD. Methods: A genetic and enzymatic characterization was obtained in all patients. FD was identified in 38 out of 65 screened subjects ( 25 females and 13 males), which belonged to 5 families identified from index cases. ECG and echocardiography was used to evaluate cardiac involvement.

Results: Cardiac involvement was present in $66 \%$ of adults and absent in all children. HVI was the most frequent abnormality observed in 56 of adults (63\% in males, $52 \%$ in females). The pre-

\section{Introducción}

La enfermedad de Fabry (EF) es un error innato del catabolismo de los glicoesfingolípidos, de herencia ligada al cromosoma $\mathrm{X}$, causado por la deficiencia de la enzima lisosomal alfa-galactosidasa $\mathrm{A}\left(\alpha-\text { gal }^{\mathrm{A}}\right)^{\mathbf{1 - 6}}$. La enzima $\alpha$-gal A es responsable de la hidrólisis de los residuos alfa-galactosil terminales de glucolípidos y glicoproteínas. Esta deficiencia ocasiona la acumulación progresiva de GB-3 en los lisosomas del glomérulo renal, las células epiteliales tubulares, las células miocárdicas, el sistema nervioso autonómico y las células de la córnea, así como del endotelio y músculo liso de las paredes vasculares 1,2,3,5.

Afecta más a los hombres que a las mujeres: se calcula que 1 en 40.000 varones tienen la enfermedad de Fabry, mientras que la prevalencia se estima en 1:80.000 a 1:117.000 nacidos vivos ${ }^{\mathbf{1}, \mathbf{4}}$. Las mujeres eran consideradas portadoras asintomáticas, pero dicho concepto ha sido abandonado en base a estudios que han demostrado valence of HVI increased with age reaching 100\% in males and $82 \%$ in females aged 40 and older. Among other findings, cardiac dilatation was observed in $26 \%$, mild mitral insufficiency in $47 \%$ and atrial fibrillation in 3 cases. No case of coronary artery disease was identified

Conclusion: Fabry's disease is more prevalent than usually suspected. Cardiac involvement is frequent, especially the presence of HVI. It should be investigated in all subjects with unexplained LVH, especially when associated to characteristic dermatologic and ophtalmologic findings. Confirmation of the diagnosis may be obtained through enzymatic and genetic studies.

Keywords: Fabry's disease, cardiac involvement, genetic lynkage.

también en ellas compromiso multiorgánico. 1,2,3,5

Las primeras manifestaciones clínicas de esta enfermedad aparecen en la niñez con crisis de dolor intenso en manos y pies, hipohidrosis, fiebre intermitente y aparición de lesiones cutáneas de localización características, denominadas angioqueratomas. La enfermedad progresa en el tiempo, apareciendo luego compromiso miocárdico, cerebrovascular e insuficiencia renal, que resulta en la

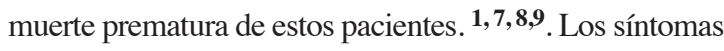
y signos cardiovasculares incluyen palpitaciones, disnea, mareos, angina de pecho y fatiga.

La acumulación de GB-3 en el corazón lleva a un incremento del grosor de la pared ventricular, con hipertrofia, que en general es concéntrica, y menos frecuentemente con afectación de ventrículo derecho. La disfunción diastólica es un hallazgo frecuente. Se ha descrito hipoquinesia inferior basal en algunos casos. ${ }^{1}$ El compromiso valvular por cambios infiltrativos es más frecuente sobre válvula mitral. Las válvulas aparecen 
engrosadas con insuficiencia de grado leve o moderado. Las arritmias más frecuentes son taquicardia paroxística supraventricular y fibrilación auricular. Los defectos en la conducción como PR corto no se asocian a un haz accesorio sino a conducción A-V acelerada. Conforme aumenta la edad aparecen bloqueos de rama, bloqueo A-V y disfunción del nódulo sinusal. Puede producirse isquemia miocárdica que en general no se relaciona a obstrucción de arterias epicárdicas, sino a un aumento de las demandas de oxigeno en el miocardio hipertrófico, asociado a disminución de densidad capilar y aumento en la presión del llenado diastólico, con la consecuente disminución de la reserva coronaria.

El Electrocardiograma y ecocardiograma son una herramienta esencial y accesible para una aproximación diagnóstica. El diagnóstico definitivo se basa en la demostración de la deficiencia severa de la actividad enzimática de la $\alpha$-galactosidasa A(DBS) y en las mujeres con el examen genético.

Desde el año 2001 se encuentra disponible la terapia de reemplazo enzimático (TRE), la cual disminuye los depósitos de GB3 en los tejidos, modificando sustancialmente el pronóstico y calidad de vida de los pacientes ${ }^{\mathbf{2 , 4 - 6}}$. La desventaja de la TRE es su alto costo. En Europa está aprobado el uso de agalsidasa alfa $(0.2 \mathrm{mg} /$ $\mathrm{kg}$ cada dos semanas) y en EEUU la utilización de agalsidasa beta ( $1 \mathrm{mg} / \mathrm{kg}$ cada dos semanas) (6), disponible actualmente en Chile. Existe, además, un estudio clínico fase III del uso de chaperonas farmacológicas en pacientes que tienen cierto tipo de mutaciones. ${ }^{\mathbf{1 0}}$

En Chile hay alrededor de 65 pacientes con enfermedad de Fabry. Estos pacientes están distribuidos principalmente en el Norte de Chile. En los últimos 3 años, en el Hospital San Pablo de Coquimbo (IV región), 6 pacientes fueron diagnosticados por presencia de lesiones en piel compatibles con angioqueratomas; en cuatro pacientes en diálisis, pesquisados durante evaluación pre transplante renal y en dos pacientes que consultaron por las lesiones asociados a síntomas neurológicos de la enfermedad. Esta situación motivó la formación de un equipo multidisciplinario para el estudio integral de los pacientes e investigar la presencia de EF en los familiares, determinar el grado de compromiso orgánico y describir las mutaciones que causan la enfermedad en estas familias y, como desafío, obtener la terapia de reemplazo enzimático para los afectados.

\section{Método}

Un Grupo interdisciplinario conformado por especialistas (dermatóloga, nefrólogo, cardióloga, oftalmóloga y neurólogo) iniciaron el estudio de estos pacientes en el Hospital San Pablo de Coquimbo. Previa lectura y firma de consentimiento informado, se elaboró el familiograma de 5 familias a partir de un caso índice con diagnóstico de EF (confirmados por DBS y/o microscopía electrónica en biopsia de piel o biopsia renal). Se encuestó a todos los familiares potencialmente afectados sobre sintomatología inherente a dicha enfermedad (trastornos de la sudoración, dolores en las extremidades inferiores o superiores, fiebre inexplicable, dolores articulares, cólicos intestinales, diarrea, aparición de manchas de color rojizo - violáceas en la piel, problemas cardíacos o renales, asma bronquial, mareos y acufenos).

Se seleccionaron 65 personas en quienes se extrajo sangre para determinación enzimática (DBS) (gota de sangre seca en papel de filtro) y genotipificación ( $5 \mathrm{ml}$ de sangre) enviadas en dos grupos al Massachusetts General Hospital (USA).

Se consideraron como adultos a los mayores de 18 años. De las 65 muestras, se confirmó la EF en 42 pacientes; dos adultos y dos menores se retiraron voluntariamente del estudio.

A los pacientes confirmados se les realizó: evaluación clínica dermatológica, neurológica, oftalmológica, nefrológica y cardiológica. Exámenes de sangre y orina (hemograma y VHS, glicemia, uremia, electrolitos plasmáticos, creatininemia, ácido úrico, perfil lipídico, proteinemia, perfil hepático, sedimento urinario, proteinuria en muestra aislada), ecografía renal, escáner de cerebro (1 paciente), biopsia de piel con microscopia electrónica (2 pacientes), ECG de 12 derivaciones, Ecocardiograma bidimensional ( Aloka 2000), Holter y test de esfuerzo (optativo). Se efectuó coronariografía en dos pacientes.

Se estableció como criterio de HVI al electrocardiograma índice de Sokolov mayor a $35 \mathrm{~mm}$ en adultos y mayor a 40 mm en niños y al Ecocardiograma septum interventricular o pared posterior mayor a $11 \mathrm{~mm}$ asociado a IMVI indexado a superficie corporal mayor a $118 \mathrm{gr} / \mathrm{m} 2$ en hombres y $104 \mathrm{gr} / \mathrm{m} 2$ en mujeres.

Se estableció como criterio de dilatación de ventrículo izquierdo un diámetro diastólico mayor a $55 \mathrm{~mm}$ y/o sistólico mayor a $37 \mathrm{~mm}$.

Electrocardiograma (ECG): En el ECG se consideraron valores normales:: FC 60-100 latidos por min; PR: 120200 mseg; QRS 70-110 mseg; QT corregido según la fórmula de Bazet (QTc B) < 450 hombres y < 470 mujeres; Índice Sokolow $<35 \mathrm{~mm}$. 
En las mediciones ecocardiográficas, según recomendaciones de la Sociedad Americana de Ecocardiografía, se evaluaron los parámetros: diámetro diastólico del ventrículo izquierdo (DDVI) 30-55 mm; septum interventricular en diástole $($ SIVD) $<11 \mathrm{~mm}$; pared posterior VI (PPVI) $<11 \mathrm{~mm}$; diámetro aurícula izquierda (DAI) 26-40 mm; índice de masa VI (IMVI) $<118 \mathrm{~g} / \mathrm{m} 2$ en hombres y < 104 en mujeres; tiempo de desaceleración mitral (TDM) 150-240 mseg; tiempo de relajación isovolumétrica mitral $>55 \mathrm{mseg}$; presión pulmonar sistólica (PPS) $<30 \mathrm{~mm} \mathrm{Hg}$. Fracción de eyección por formula de Teichholz: desde 55 a $76 \%$.

\section{Resultados}

Se diagnosticaron 38 pacientes con EF, 25 mujeres $(65.8 \%)$ y 13 hombres (34.2\%). Ocho pacientes eran niños y 30 adultos (11 hombres - 19 mujeres).

La edad promedio en niños fue de 12 años ${ }^{\mathbf{9}, 16}$. La edad promedio en adultos fue de 39 años $\mathbf{1 8}$ - $\mathbf{7 4}$, en ambos grupos con predominio femenino.

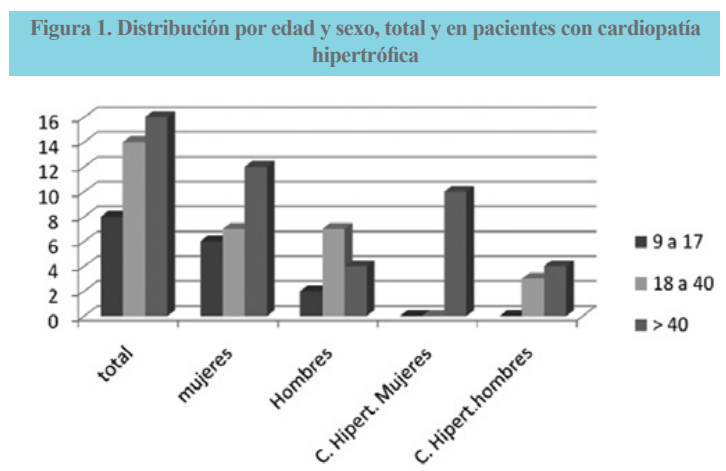

En mayores de 40 años el $100 \%$ de hombres y el $82 \%$ de las mujeres están afectados. La edad promedio de los pacientes con $\mathrm{CH}$ fue de 38 años (28-48) en los hombres y en las mujeres de 57 años (41-74).
La mutación encontrada fue P259R en casi todos los pacientes, salvo un hombre con mutación W81X, considerada probablemente mutación de novo. Un $50 \%$ de las mujeres presentó un dosaje enzimático (DBS) normal, en ellas el diagnóstico fue confirmado por estudio molecular; en cambio, en el 100\% de los hombres la DBS fue bajo rango normal.

El compromiso cardiológico estuvo presente en el $66 \%$ de los pacientes adultos, no encontrándose hallazgos cardiológicos patológicos en los niños.

La cardiopatía hipertrófica $(\mathrm{CH})$ fue el hallazgo cardiológico más frecuente, presente en $56 \%$ de los adultos ( 7 hombres y 10 mujeres) ( fig 2). Si consideramos la prevalencia por sexo, el $63 \%$ de los hombres y el 52\% de las mujeres presentaban hipertrofia cardíaca. En mayores de 40 años se observó que el $100 \%$ de hombres y el $82 \%$ de las mujeres estaban afectados, observándose mayor grado de hipertrofia conforme aumenta la edad. El promedio del SIV al ecocardiograma fue de $15 \mathrm{~mm}$, y de $17 \mathrm{~mm}$ si consideramos sólo a los mayores de 45 años. La edad promedio de los pacientes con $\mathrm{CH}$ fue de 38 años (28-48) en los hombres y en las mujeres de 57 años (41-74). La HVI al ecocardiograma es en general simétrica, con aumento de refringencia en la zona central (foto 1), no evidenciándose aumento del gradiente en el tracto de salida de ventrículo izquierdo en estos pacientes. En algunos casos se observó aumento del gradiente en el tracto de salida de ventrículo izquierdo.

Nueve pacientes tenían HVI al ECG, todos ellos con HVI al ecocardiograma. En cambio, sólo 53\% de los pacientes con HVI al ecocardiograma la tenían también en el ECG. De los pacientes sin HVI al ECG, 35\% presentaba Bloqueo completo o incompleto de rama derecha. Sólo un paciente tenía ECG normal.

La cardiopatía dilatada estuvo presente en 8 pacientes adultos (26\%) sin diferencias por sexo, 7 de los cuales

Figura 2. Ecocardiograma ilustrativo de HVI, con septum de $21 \mathrm{~mm}$ en un paciente con Enfermedad de Fabry
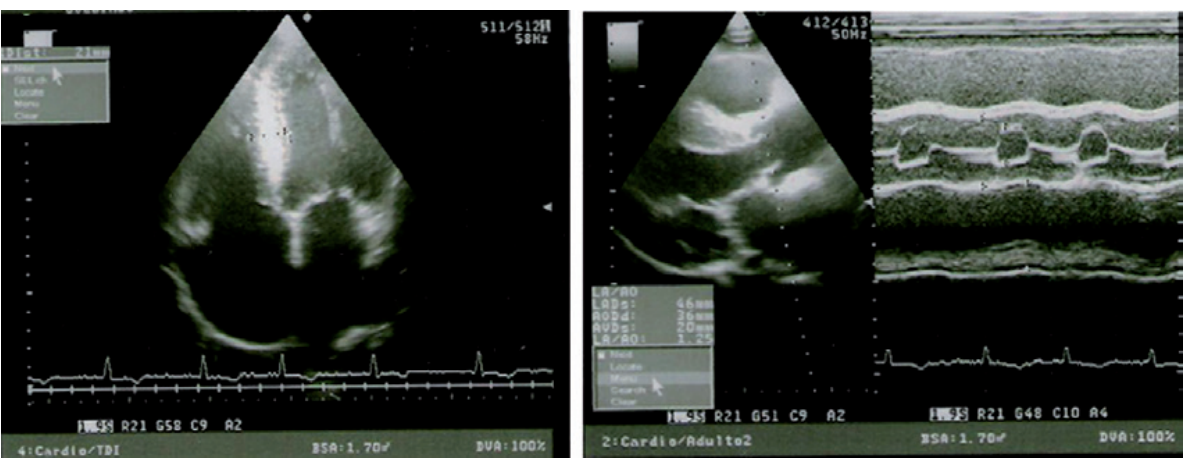

Ecocardiograma en un paciente con EF: Hipertrofia ventricular izquierda con grosor septal de $21 \mathrm{~mm}$ 


\begin{tabular}{|l|lll|}
\hline \multicolumn{4}{c}{ Tabla 1. Compromiso cardíaco en 30 adultos con } \\
Enfermedad de Fabry \\
\hline Pacientes adultos & 30 & 11 & 19 \\
HVI & 17 & 7 & 10 \\
Cardiopatía dilatada & 7 & 3 & 4 \\
Pr corto & 7 & 0 & 7 \\
Insuficiencia mitral leve & 14 & 5 & 9 \\
Arritmias & 4 & 1 & 3 \\
BCRD/ BIRD & 6 & 0 & 6 \\
Bloqueo A-V completo & 1 & 1 & 0 \\
\hline
\end{tabular}

estaban asociados a $\mathrm{CH}$ y cuatro de ellos en diálisis. El único paciente con cardiopatía dilatada sin hipertrofia asociada es el más joven del grupo (21 años) y presenta la mutación de novo.

La fracción de eyección estuvo disminuida en 4 pacientes, tres mujeres y 1 hombre, todos ellos con dilatación e hipertrofia ventricular.

Se observó insuficiencia valvular mitral leve en 47\%,con predominio femenino (55\%) y PR corto en 7 mujeres (18\%). Entre las arritmias se observó: TPSV 1 mujer (2.6 $\%$, Fibrilación auricular en 2 mujeres y en 1 hombre. BCRD: en 3 mujeres, BIRD: en 3 mujeres, BAV completo en 1 hombre (2.6\%), que requirió marcapaso definitivo.

Dos pacientes con angina de pecho tenían coronarias angiográficamente normales. No se registraron eventos coronarios.

Entre los hallazgos extracardíacos destacó: depósitos corneales en 28 pacientes (73\%) con predominio femenino (78\%), angioqueratomas en 12 pacientes (31\%) masculinos (ilustrado en la Figura 3) . Hubo Insuficiencia Renal Crónica en 5 pacientes, 3 en Diálisis y uno trasplantado, todos ellos con compromiso cardíaco.

\begin{tabular}{|c|c|c|c|}
\hline & total & mujeres & hombres \\
\hline pacientes & 38 & 25 & 13 \\
\hline cornea verticillata & 28 & 19 & 9 \\
\hline acroparestesias & 14 & 6 & 8 \\
\hline angioqueratomas & 12 & 1 & 11 \\
\hline insuf Renal & 5 & 2 & 3 \\
\hline
\end{tabular}

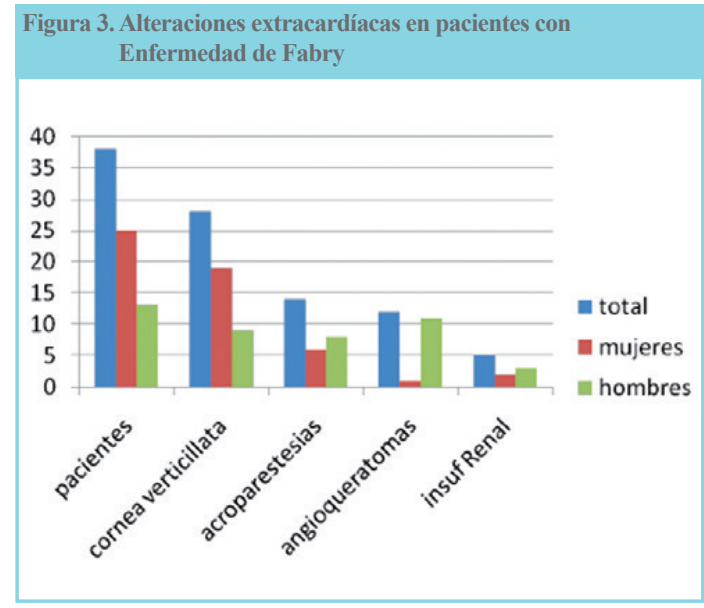

Las acroparestesias se manifestaron desde los 9 años y estuvieron presentes en el $67 \%$ de los pacientes, con predominio masculino. (Tabla 2).

\section{Discusión}

La EF es una patología rara, subdiagnosticada y potencialmente letal que afecta a ambos sexos. Por razones desconocidas, en nuestra comuna (215.109 habitantes) se encuentra con mayor frecuencia (1/ 6000 habitantes). La existencia de TRE obliga a identificar precozmente los síntomas y signos sugestivos de la enfermedad para realizar un diagnóstico y tratamiento precoz.

La afectación cardíaca es similar a la reportada internacionalmente y está presente en gran parte de los adultos, observándose similar incidencia en ambos sexos, con aparición más tardía en mujeres, en las cuales el grado de hipertrofia es tan severo como en los hombres.

El hallazgo de hipertrofia ventricular izquierda al ecocardiograma tuvo una importante correlación con el electrocardiograma, salvo en los pacientes con bloqueo completo de rama derecha.

De las pacientes con $\mathrm{PR}$ corto, solo una no tiene $\mathrm{CH}$, y en

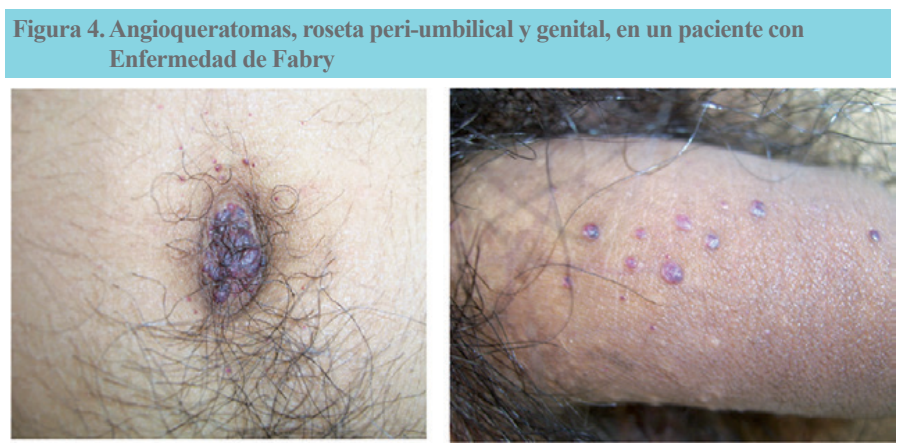

Angioqueratomas: roseta peri umbilical y genital en un paciente con Enfermedad de Fabry gentileza: Dra Carmen Varas). 
dos pacientes el PR corto fue hallado en ECG previos ya que actualmente están en fibrilación auricular.

Los cuatro pacientes con IRC terminal presentaron Cardiopatía hipertrófica y dilatada, en cambio sólo el 30\% de los pacientes con $\mathrm{CH}$ presentaban disfunción renal.

Antes de este estudio a algunos de estos pacientes ya se les había realizado el diagnóstico de miocardiopatía hipertrófica, pero no se había sospechado la EF. De allí la importancia de tenerla presente en el diagnóstico diferencial de Hipertrofia ventricular izquierda de origen desconocido, no hipertensiva, en pacientes jóvenes, apoyados por los síntomas extracardíacos. También sospecharla en aquellos pacientes con $\mathrm{CH}$ en diálisis cuya causa del daño renal no esté establecida.

Es de suma importancia apoyarse de la evaluación dermatológica y oftalmológica, la cual nos permite una aproximación diagnóstica antes de solicitar los estudios enzimáticos y moleculares. También es importante el estudio de los familiares ya que en el caso de los pacientes hombres todas sus hijas estarán comprometidas, comparado con sólo el $50 \%$ en las mujeres.

Creemos que todavía existen en Chile, y sobre todo en el norte, pacientes con EF aun no diagnosticados, ya que estas familias son muy numerosas y, de acuerdo a la mutación encontrada, pertenecen a un antepasado común.

Agradecimientos: A la empresa farmacéutica Genzyme por la posibilidad de financiar la realización de determinación enzimática y el estudio molecular a nuestros pacientes.

\section{Referencias:}

1. KAMPMANN C, LINHART A, BAEHNER F, PALECEK T, WIETHOFF CM, MIEBACH E, et al. Onset and progression of the Anderson-Fabry disease related cardiomyopathy. Int J Cardiol 2008; 130: 367-73.

2. DESNICK RJ, IOANNOU YA, ENG CM. a-Galactosidase a deficiency: Fabry disease. In: Scriver CR, Beaudet AL, Sly WS, editors. The metabolic and molecular bases of inherited disease. New York: McGraw-Hill; 2001; 3733-74.

2. THADHANIR,WOLFM,WESTML,TONELLIM,RUTHAZER R, PASTORES GM, et al. Patients with Fabry disease on dialysis in the United States. Kidney Int 2002; 61: 249-255.

3. ALROY J, SABNIS S, KOPP JB. Renal pathology in Fabry disease. J Am Soc Nephrol 2002; 13: S134-S138.

4. ORTIZ A, OLIVEIRA JP, WANNER C, BRENNER BM, WALDEK S, WARNOCK DG. Recommendations and guidelines for the diagnosis and treatment of Fabry nephropathy in adults. Nat Clin Pract Nephrol 2008; 4: 327-336.

5. TAHIR H, JACKSON LL, WARNOCK DG. Antiproteinuric therapy and Fabry nephropathy: sustained reduction of proteinuria in patients receiving enzyme replacement therapy with agalsidase-beta. J Am Soc Nephrol 2007; 18: 2609-2617.
6. WILCOX WR, BANIKAZEMI M, GUFFON N, WALDEK S, LEE P, LINTHORST GE, et al. Long-term safety and efficacy of enzyme replacement therapy for Fabry Disease. Am J Hum Genet 2004; 75: 65-74.

7. MACDERMONT KD, HOLMES A, MINERS AH. ANDERSON-FABRY DISEASE: clinical manifestations and impact of disease in a cohort of 98 hemizygous males. J Med Genet 2001; 38: $750-60$

8. KAMPMANN C, LINHART A, BAEHNER F, PALECEK T, WIETHOFF CM, MIEBACH E, et al. Onset and progression of the Anderson-Fabry disease related cardiomyopathy. Int J Cardiol 2008; 130: 367-73.

9. KAMPMANN C, BAEHNER F, RIES M, BECK M. Cardiac involvement in Anderson-Fabry disease. J Am Soc Nephrol 2002; 13: S147-9.

10. FAN JQ, ISHII S. Active site-specific chaperone therapy for Fabry Disease. FEBS J 2007; 274: 4962-71.

11. LINHART A, KAMPMANN C, ZAMORANO JL, SUNDERPLASSMANN G, BECK M, MEHTAA, et al. Cardiac manifestations of Anderson-Fabry disease: results from the International Fabry Outcome Survey. Eur Heart J 2007; 28: 1228-35.

12. LINHART A, ELLIOTT PM. The heart in Anderson-Fabry disease and other lysosomal storage disorders. Heart 2007; 93: 528-35. 